\title{
XIII. Description of a thermometer for determining minute differences of temperature
}

\author{
Marshall Hall M.D. F.R.S.
}

To cite this article: Marshall Hall M.D. F.R.S. (1836) XIII. Description of a thermometer for determining minute differences of temperature, Philosophical Magazine Series 3, 8:43-44, 56-58, DOI: $10.1080 / 14786443608648798$

To link to this article: http://dx.doi.org/10.1080/14786443608648798

曲 Published online: 21 Aug 2009.

Submit your article to this journal $₫$

Џ Article views: 2

Q View related articles $₫$ 
the magnet, and also on its length. Biot has shown that in a steel wire 24 inches long, and properly magnetized, the pole is an inch and a half from its extremity, and that this distance diminishes with every diminution in the length of the magnet*. The centre of parallel forces or the pole of a magnet is similar to the centre of gravity of a body. In the one case the effect is the same as if all the matter of which the body is composed were concentrated in the centre of gravity, in the other the effect is the same as if the difference between the sum of all the attractive and repulsive forces were concentrated in the pole. Now, in the case of the mulual attraction of bodies, our measurements are always taken between the centres of gravity; in the case of magnetic attractions the distances of the magnets are, in fact, the distances between the poles.

Let the distance of the poles of the magnets when in apparent contact be called2, as in fig. (1.), and then separated by an interval of 1 , as in fig. (2.), and by intervals of 2 . as in

(2.)

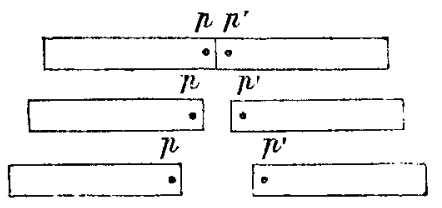
fig. (3.)

Then the distances between the centres of force in these three positions are $2,3,4$. Hence if the law of the inverse squares of the distances, investigated by Coulomb, be the real law of action, the attractive forces will be inversely as $2^{2}, 3^{2}, 4^{2}$, that is, as $\frac{1}{4}, \frac{1}{9}, \frac{1}{16}$; but $\frac{1}{9}$ is nearly the half of one fourth, and $\frac{1}{16}$ nearly the half of $\frac{1}{9}$, as Mr. Fox found by actual experiment. These experiments then, instead of leading to a new law of action, afford a beautiful illustration of that law which universally prevails whenever we have matter acting on matter by attractive or repulsive forces.

XIII. Description of a Thermometer for determining minute Differences of Temperature. By Marshall Hali, M.D., F.R.S.\&. $†$

IN pursuit of the theory of the inverse ratio of the respiration and of the irritability in the animal kingdom, announced in a late volume of the Philosophical Transactions $\$$, I have found it absolutely necessary to determine the minute

* Biot, Traité de Physique, tom. iii. p. 90.

+ Communicated by the Author.

In abstract of Dr. Marshall Hall's paper on this subject will be found in Phil. Mag. and Annals, N.S. vol. xi, p. 453.-Edrt. 
differences of temperature which exist in animals of the same class. In pursuing this inquiry, I soon discovered that it was essential to devise other instruments than those in ordinary use.

It was easy by enlarging the bulb and by selecting a tube of extremely fine calibre, to render the common thermometer capable of more minute indications. But it was impossible to carry this change beyond a certain degree, the augmented length of the instrument becoming highly inconvenient.

In order to obviate this difficulty, I devised the instrument which I am now about to describe.

The form of this instrument is represented in the accompanying outline. The relative size of the bulb and calibre of the tube is such that the tenth part of a degree occupies a considerable space upon the scale. The entire scale consists of ten degrees. At the upper part of the thermometric tube a small bulb is blown, which I shall designate the reservoir; it is turned forwards so as to remain at a right angle with the tube.

The bulb and the tube are filled with mercury, and a little of that fluid is included in the reservoir, when the whole is hermetically sealed.

When an experiment is to be made, the mercury in the tube is to be brought into contact with the mercury in the reservoir, by placing the in-

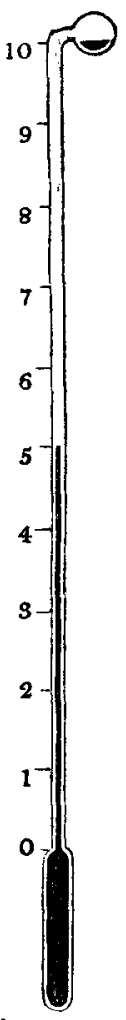
strument horizontally, with the reservoir upwards, in water of a sufficient temperature.

I will now suppose that I wish to try the comparative temperature of the swallow which shuns, and the sparrow which abides, the rigours of our winter. The thermometer is removed from the water at the temperature of $110^{\circ}$ Fahr., and placed upright. The contiguity of the mercury in the tube with the mercury in the reservoir being broken, the highest point in the scale will represent that degree, viz. $110^{\circ}$. The lowest will consequently be the 100 th degree. The entire scale is one of six degrees between these extremes, each degree being divided into tenths.

The same plan is adopted for any other part of the scale.

We have thus an instrument of the usual size, capable of measuring the tenths of a degree of temperature, at any part of the scale. It only requires the addition of a common thermometer to afford the extreme limit of the magnified scale.

Thivd Series. Vol. 8. No. 43. Jan. 1836. 
I may be permitted to add, that the temperature of an animal indicated by such a thermometer compared with that of the medium in which it is placed, affords a near approximation to the degree of respiration, and, inversely, of the irritability of the muscular fibre.

\footnotetext{
LXV. Proceedings of Learned Societies.

OFFICIAL REPORT OF THE PROCEEDINGS OF THE BRITISH ASSOCIATION FOR THE ADVANCEMENT OF SCIENCE, AT THE DUBLIN MEETING, AUGUST 1835.

Communicated by the Council and Secretaries.

[Continued from vol. vii. p. 513.]

Notices and Alstracts of Miscellaneous Communications to the Sections, continued.

MEDICAI SCIENCE,--continued.

Experimental Inquiry into the different Offices of Lacteals, Lymphatics, and Veins in the Function of Absorption. By P.D. HANDYSIDE, M.D.
}

TTHE author's general position is thus stated: "The lacteals, lymphatics, and veins are endowed each with a peculiar office in the general functions of absorption; for example, 1. The lacteals are those vessels which absorb the aliment which is necessary for maintaining the nutrition and increase of the body, and exercise the property of refusing entrance to all other matters; 2 . The lymphatics absorb the elements of the body upon their becoming useless or noxious, so as by their final discharge from the system to make room for the deposition of new matter, and these vessels possess no absorbing power over any substances foreign to the system; 3 . The veins not only return to the heart the blood after that fluid has fulfilled the object of its diffusion over the system, but enjoy the office of receiving into the animal system by absorption various foreign matters which may be brought into contact with their orifices.

In support of these views the author presents a short review of results obtained by various eminent anatomists and physiologists.

The following is the order of the subjects discussed:

Lacteals.-Their distention after a full meal,---their condition as observed in living animals; - -effects of ligatures on the thoracic ducts of horses.

Lymphatics,-Anatomical origin of,-analogy of lymphatics and lacteals,-exact resemblance of the lymph prior to its absorption to that found in the lymphatic vessels,-absence of lymphatics in vegetables, - no proof afforded by examination of lymph that lymphatics serve as the channel through which foreign matters gain entrance into the system,-no communication between lymphatics and veins except through the great lymphatic trunks.

Veins.-Analogy between the anatomy and disposition of the veins of animals and the vessels corresponding to these in plants, favours the doctrine of venous absorption. 